\title{
Analysis of Screening Results of Four Common Infectious Diseases in One Stomatology Hospital
}

\author{
Peng Sun1,2*, Haili Yu' ${ }^{2 *}$ Meiling Yao², Xiaolin Xu${ }^{1 \#}$ \\ ${ }^{1}$ Department of Orthodontics, Stomatological Hospital of Yantai Affiliated to Binzhou Medical College, Yantai, China \\ ${ }^{2}$ Department of Medical, Stomatological Hospital of Yantai Affiliated to Binzhou Medical College, Yantai, China \\ Email: "drsun1983@163.com, "best32@163.com
}

How to cite this paper: Sun, P., Yu, H.L. Yao, M.L. and Xu, X.L. (2020) Analysis of Screening Results of Four Common Infectious Diseases in One Stomatology Hospital. Journal of Biosciences and Medicines, $\mathbf{8}$, 96-102.

https://doi.org/10.4236/jbm.2020.84008

Received: March 11, 2020

Accepted: April 12, 2020

Published: April 15, 2020

Copyright (c) 2020 by author(s) and Scientific Research Publishing Inc. This work is licensed under the Creative Commons Attribution International License (CC BY 4.0).

http://creativecommons.org/licenses/by/4.0/

\begin{abstract}
Objective: To understand the status and characteristics of hepatitis $\mathrm{B}$, hepatitis $\mathrm{C}$, syphilis and HIV antibody screening in one dental hospital, and to provide scientific basis for the prevention and treatment of common infectious diseases in dental medical institutions. Methods: Collect all samples of patients screened for hepatitis B, hepatitis C, syphilis and HIV from one stomatological hospital in 2018-2019, and make statistics on the distribution of common infectious diseases among patients in stomatology, such as age, gender, type of treatment, department of treatment, etc. Results: The positive rate of hepatitis B was $2.27 \%, 39.42 \%$ in males and $60.58 \%$ in females. It was mainly detected by outpatient department, accounting for $87.5 \%$. The age was concentrated in 30 - 60 years old, accounting for $77.88 \%$. It was mainly distributed in oral and maxillofacial surgery, orthodontics and implant, accounting for $82.69 \%$. The positive rate of hepatitis C was $0.1 \%, 33.33 \%$ in men and $66.67 \%$ in women. It was mainly detected by outpatient department, accounting for $83.33 \%$. The age is concentrated in $20-50$ years old, accounting for $100 \%$. It is mainly distributed in oral and maxillofacial surgery, orthodontics and implant, accounting for $100 \%$. The positive rate of syphilis was $0.18 \%, 33.33 \%$ for men and $66.67 \%$ for women. It was mainly detected by outpatient department, accounting for $83.33 \%$. The age is concentrated in 30 - 50 years old, accounting for $100 \%$. It was mainly distributed in oral and maxillofacial surgery, orthodontics and implant, accounting for $83.33 \%$. HIV was not detected. Conclusion: The positive detection rate of common infectious diseases in the stomatological hospitals is generally lower than the average level. It is mainly distributed in the department of implant, oral and maxillofacial surgery and orthodontics. The highest positive detection rate of common infectious diseases is hepatitis B which is $2.27 \%$, and the lowest is HIV which is 0 .
\end{abstract}




\section{Keywords}

Stomatology, Infectious Diseases, Screening

\section{Introduction}

With the development of society, people's living standards are also improved, and the demand for oral health care is increasing. However, due to the increasingly serious epidemic trend of diseases transmitted by body fluids, blood, droplets, etc., and the complicated operation process of oral medicine, the accidental injury of sharps will lead to direct contact between body fluids and blood, and the bacterial aerosols containing plaque, debris of dental stones, dental materials, blood, saliva, and unsterilized dental water generated by the high-speed rotation of the rapid turbine phone [1] are the media of disease transmission [2]. In view of the particularity of stomatology, people pay more and more attention to the cross infection in oral therapy. Hepatitis B, hepatitis C, syphilis, HIV and other diseases are common through body fluids, blood and other diseases; stomatology should pay special attention to them. Therefore, by collecting all samples of patients screened for hepatitis B, hepatitis C, syphilis and HIV from one stomatology hospital in 2018-2019, this study makes statistics on the age, gender, department and other distribution of common infectious diseases in stomatology patients, so as to provide scientific basis for the prevention and treatment of common infectious diseases in stomatology.

\section{Samples and Methods}

\subsection{Sample Selection}

All the patients who were tested for hepatitis B, hepatitis C, syphilis and HIV antibody from 2018 to 2019 were selected from one stomatological hospital.

\subsection{Research Method}

Extract the age, gender, visit category, visit department, screening results and other information of this part of patients, make classified statistics on the information, and make statistics on the age, gender, visit category, visit department and other distribution of common infectious diseases in patients of Stomatology.

\section{Results}

\subsection{General Situation of Screening}

There were 4582 samples of patients who were tested for hepatitis B in stomatology hospital, 104 positive samples were detected, the positive rate was $2.27 \%$. A total of 4404 patients were tested for hepatitis C, 6 of them were positive, the positive rate was $0.1 \%$. A total of 3309 syphilis patients were tested, 6 of them were positive, the positive rate was $0.18 \%$. A total of 4582 patients were tested for 
$\mathrm{HIV}$, and 0 positive samples were detected, with a positive rate of $0 \%$.

\subsection{Age Distribution}

The age of hepatitis B screening positive patients in stomatology hospital is mainly concentrated in 30 - 60 years old, accounting for $77.88 \%$, among which the number of 40 - 50 years old positive patients is the most, accounting for $27.88 \%$. The age of hepatitis C screening positive patients is concentrated in 20 50 years old, accounting for $100 \%$, of which the number of 20 - 30 years old positive patients is the most, accounting for $50 \%$. The age of syphilis screening positive patients is concentrated in 30 - 50 years old, accounting for $100 \%$, of which the number of 30 - 40 years old positive patients is the most, accounting for $66.66 \%$. HIV was not detected. See Table 1 for details.

\subsection{Gender Distribution}

The gender of hepatitis $B$, hepatitis $C$ and syphilis screening positive patients in stomatology hospital is more than that of men. The proportion of women in the total number of positive patients in three infectious diseases is $60.58 \%, 66.67 \%$ and $66.67 \%$ respectively. HIV was not detected. See Table 2 for details.

\subsection{Visit Category Distribution}

The positive patients of hepatitis $\mathrm{B}$, hepatitis $\mathrm{C}$ and syphilis were mainly detected in outpatient department, More than $80 \%$ of the patients were positive, see $\mathrm{Ta}$ ble 3 for details.

Table 1. Age distribution percentage of four common infectious diseases in stomatology (\%).

\begin{tabular}{ccccc}
\hline $\begin{array}{c}\text { Age/year } \\
\text { Disease types }\end{array}$ & hepatitis B & hepatitis C & syphilis & HIV \\
\hline $10-20$ & 3.85 & 0 & 0 & 0 \\
$20-30$ & 14.42 & 50.00 & 0 & 0 \\
$30-40$ & 18.27 & 16.67 & 66.66 & 0 \\
$40-50$ & 27.88 & 16.67 & 16.67 & 0 \\
$50-60$ & 20.19 & 16.66 & 16.67 & 0 \\
$60-70$ & 11.54 & 0 & 0 & 0 \\
$70-80$ & 3.85 & 0 & 0 & 0 \\
\hline
\end{tabular}

Table 2. Sex distribution percentage of four common infectious diseases in stomatology (\%).

\begin{tabular}{ccccc}
\hline $\begin{array}{c}\text { Gender } \\
\text { Disease types }\end{array}$ & hepatitis B & hepatitis C & syphilis & HIV \\
Male & 39.42 & 33.33 & 33.33 & 0 \\
Female & 60.58 & 66.67 & 66.67 & 0 \\
\hline
\end{tabular}


Table 3. Percentage distribution of four types of common infectious diseases in stomatology (\%).

\begin{tabular}{ccccc}
\hline $\begin{array}{c}\text { Visiting categories } \\
\text { Disease types }\end{array}$ & hepatitis B & hepatitis C & syphilis & HIV \\
\hline $\begin{array}{c}\text { Outpatient Department } \\
\text { Inpatient Department }\end{array}$ & 85.58 & 83.33 & 83.33 & 0 \\
\hline
\end{tabular}

\subsection{Department Distribution}

The patients with hepatitis $B$, hepatitis $C$ and syphilis were mainly detected in the departments of oral and maxillofacial surgery, periodontal surgery, orthodontics and implant department. The positive patients accounted for more than $90 \%$ of the total positive patients, see Table 4 for details.

\section{Discussion}

The outpatient department of stomatology is a department that operates examination, diagnosis and treatment in the same clinic at the same time. It is a high-risk department for hospital infection. The main reasons are as follows: First of all, the patients' blood and saliva are often contacted in the process of diagnosis and treatment in stomatology, and there are pathogenic microorganisms in the blood and saliva; Secondly, sharp instruments are often used in stomatological treatment, which is easy to cause occupational exposure and hospital infection; Finally, the instruments used in stomatology, such as turbo mobile phone and ultrasonic dental cleaner, will produce a large number of aerosols when they work. The aerosols contain pathogenic microorganisms, which will pollute a large area of space around the work area [3] [4]. Therefore, patients and medical staff are the high-risk groups who are infected with common infectious diseases such as hepatitis B, hepatitis C, syphilis, HIV, etc. This study aims at the screening of hepatitis B, hepatitis C, syphilis, HIV and other common infectious diseases in stomatology, to understand the carrying situation of common infectious diseases in stomatology patients, and to provide clinical guidance for prevention and control of cross infection in stomatology.

China is a big country of hepatitis B, nearly $1 / 3$ of the 350 million hepatitis B carriers in the world are Chinese, which is an important infectious disease that seriously threatens the health of Chinese people [5]. This study [Tables 1-4] showed that the positive rate of hepatitis B was $2.27 \%$ in the patients of stomatology department, more women than men, mainly detected by the outpatient department, the age was mainly concentrated in 30 - 60 years old, mainly distributed in the departments of oral and maxillofacial surgery, orthodontics and implant department. According to the research of Ruibo Duan and others, from 1992 to 2004, the carrying rate of hepatitis B virus in China after vaccination was $8.0 \%$, while from 2005 to 2016 , the carrying rate of hepatitis B virus dropped to $6.0 \%$ [6]. To analyze the reasons for the decrease of hepatitis B virus carrying rate is related to the improvement of people's health care awareness and the 
Table 4. Distribution percentage of four common infectious diseases departments in stomatology (\%).

\begin{tabular}{ccccc}
\hline $\begin{array}{c}\text { Visiting Department } \\
\text { Disease types }\end{array}$ & hepatitis B & hepatitis C & syphilis & HIV \\
\hline Maxillofacial surgery & 17.30 & 16.67 & 16.67 & 0 \\
Periodontist & 8.65 & 0 & 16.66 & 0 \\
Orthodontics & 14.42 & 33.33 & 16.67 & 0 \\
Implant Department & 52.88 & 50.00 & 50.00 & 0 \\
Other departments & 8.65 & 0 & 0 & 0 \\
\hline
\end{tabular}

popularization of vaccines. The main routes of transmission of hepatitis B include body fluid transmission, contact transmission, mother to child transmission, sexual transmission, iatrogenic transmission and droplet transmission [7]. The department of stomatology, especially the department using turbines and sharp instruments, should strengthen the screening of patients, take protective measures during the operation, disinfect the environment and deal with medical instruments after the operation.

This study [Tables 1-4] showed that the positive rate of hepatitis $C$ was $0.1 \%$, more women than men. It was mainly detected in outpatient department, with the age concentrated in 20 - 50 years old, mainly distributed in oral and maxillofacial surgery, orthodontics and implant department. The main reason why there are more women in the study sample is that there are more women than men, the ratio of men to women is about 1:2, so the detection rate of women in the positive sample is also higher than that of men. Since most people have no obvious clinical symptoms after hepatitis $\mathrm{C}$ infection, and there is no hepatitis $\mathrm{C}$ vaccine at present, the key measures to prevent and treat hepatitis $\mathrm{C}$ are early detection, early diagnosis and early treatment. At present, only orthodontics and surgery departments routinely screen infectious diseases, However, in order to reduce the incidence of hospital cross infection, it is necessary to carry out routine screening for patients who need treatment such as turbines and dental cleaners.

Syphilis is a kind of chronic infectious disease caused by Treponema pallidum infection, mainly transmitted by sex, blood and mother to child. The results showed that the positive rate of syphilis was $0.18 \%$ in the patients of stomatology department, more women than men. It is mainly distributed in the departments of oral and maxillofacial surgery, orthodontics and implant. According to the study of Juan Cheng et al. [8], the analysis of syphilis epidemiology shows that syphilis patients are mainly concentrated in the age group of 20 - 49 years old. The main reason is that the group has a more active sexual life, which is basically consistent with the results of this study, However, the positive rate of syphilis in the dental patients investigated in this study is significantly lower than the average level [9], which may be related to regional differences or insufficient sample size. In the future, this study will increase the sample size and reduce the error. 
No HIV positive samples were detected in this study [Tables 1-4], and the positive rate was lower than the average level and general hospital, which was consistent with the results of Jianfen Ding et al. [10], the analysis of the causes is not only related to the lower capacity and ability of the outpatients and inpatients of oral diseases in the stomatological specialized medical institutions, but also related to the cognition degree of the medical staff of the stomatological specialized medical institutions on AIDS, the ability of diagnosis and treatment of HIV related oral symptoms and the number of oral diseases for screening infectious diseases. Therefore, it is necessary to strengthen the knowledge of HIV, especially oral representation, and strengthen the importance of disease screening. At the same time, we should try our best to screen and report infectious diseases for patients with oral manifestations.

\section{Conclusion}

In conclusion, the positive detection rate of common infectious diseases in the stomatological hospitals is generally lower than the average level. It is mainly distributed in the department of implant, oral and maxillofacial surgery and orthodontics. The highest positive detection rate of common infectious diseases is hepatitis B which is $2.27 \%$, and the lowest is HIV which is 0 . This study suggests that we should strengthen the screening of infectious diseases in dental pulp department, prosthetic department and other departments. These patients may have carriers of infectious diseases, which is the potential risk of cross infection in stomatology.

\section{Conflicts of Interest}

The authors declare no conflicts of interest regarding the publication of this paper.

\section{References}

[1] Micik, R.E., Miller, R.L., Mazzarella, M.A. and Gunnar, R. (1969) Studies on Dental Aerobiology. I. Bacterial Aerosols Generated during Dental Procedures. Journal of Dental Research, 48, 49-56. https://doi.org/10.1177/00220345690480012401

[2] Hallier, C., Williams, D.W., Potts, A.J.C. and Lewis, M.A.O. (2010) A Pilot Study of Bioaerosol Reduction Using an Air Cleaning System during Dental Procedures. British Dental Journal, 209, 408-409. https://doi.org/10.1038/sj.bdj.2010.975

[3] Chen, W.P. and Huang, S.D. (2015) Preventive Measures of Environmental Infection Transmission in Stomatology Department. Chinese Journal of Practical Dentistry, 8, 56-60.

[4] Chen, W.P. and Huang, S.D. (2019) Reduce the Spread of infection Microorganisms in the Diagnosis and Treatment of Stomatology. Modern Hospital, 19, 330-332.

[5] Ji, Z.H. (2016) Study on Risk Factors and S Gene Characteristics of New Hepatitis B Infection in Northwest China. The Fourth Military Medical University, Xi'an.

[6] Duan, R.B. (2018) Meta Analysis of the Characteristics of HBV Carrying Rate in China in Recent 25 Years. Shanxi Medical University, Jinzhong. 
[7] Mikhailov, M.I., Gomberg, M.A., Dolzhanskaya, N.A. and Koubanova, A.A. (2002) Significance of Sexual Route of Transmission of Hepatitis B and C in Russia. International Journal of STD \& AIDS, 13, 9-11.

https://doi.org/10.1258/095646202762226083

[8] Cheng, J., Duan, H.Y. and Li, A.X. (2012) Analysis of the Current Situation of Syphilis Epidemiology and Diagnosis and Treatment. Infectious Disease Information, 25, 58-60.

[9] Zheng, Y.R., Li, Z.P., Liang, H.J., Huang, B.Q., Tian, Y. and Xiao, S.Y. (2010) Analysis of Syphilis Screening Results of 1226507 Unpaid Blood Donors in Guangzhou. Journal of Tropical Medicine, 10, 469-470.

[10] Ding, J.F., Qiu, J. and Shen, S.M. (2016) Analysis of HIV Antibody Screening in 9 Years in Peking University Dental Hospital. Shanghai Stomatology, 25, 97-100. 